\title{
STEINER MINIMAL TREE FOR POINTS ON A CIRCLE
}

\author{
D. Z. DU, F. K. HWANG AND S. C. CHAO
}

\begin{abstract}
We show that the Steiner minimal tree for a set of points on a circle is the shortest path connecting them if at most one distance between two consecutive points is "large". We prove this by making an interesting use of the Steiner ratio $\rho$ which has been well studied in the literature.
\end{abstract}

1. Introduction. Let $A$ denote a given set of $n$ points in the Euclidean plane. A Steiner minimal tree for $A$ is the shortest network (clearly, it has to be a tree) interconnecting $A$. Junctions of the network which are not in $A$ are called Steiner points. A tree connecting $A$ without using any Steiner points is called a spanning tree and a shortest spanning tree is called a minimal spanning tree. Let $L_{S}(A)$ and $L_{M}(A)$ denote the lengths of a Steiner minimal tree and a minimal spanning tree for $A$, respectively. Define the Steiner ratio $\rho=\inf _{A} L_{S}(A) / L_{M}(A)$. Gilbert and Pollak [4] conjectured that $\rho=\sqrt{3} / 2$ and it has recently been proved [2] that $\rho \geq .8$.

While efficient algorithms [6, 7] exist for constructing minimal spanning trees, the problem of constructing Steiner minimal trees for general set of points is known [3] to be $N P$-complete. However, efficient algorithms may exist for point-sets with special structures. In this paper we study the case that $A$ is a set of points on a circle with at most one "large" distance between two consecutive points. We prove that a minimal spanning tree for $A$ (the tree turns out to be a path) is also a Steiner minimal tree for $A$ by making an interesting use of the Steiner ratio.

2. Some preliminary results. A path $B_{1} B_{2} \cdots B_{n}$ will be called a Steiner path if it is convex and all subtending angles are $120^{\circ}$. Let $d(x, y)$ denote the distance between two points $x$ and $y$. The following two lemmas can be easily verified by elementary geometry. We state them without proofs.

LEMMA 1. Suppose that $B_{1} B_{2} \cdots B_{n}, n \leq 1$, is a Steiner path such that $d\left(B_{i}, B_{i+1}\right) \leq a$ for $i=1, \ldots, n-1$. Then $d\left(B_{1}, B_{n}\right) \leq 2 a$.

LEMMA 2. Let $W$ be any point in the triangle $\triangle X Y Z$. Then

$$
d(X, W) \leq \max \{d(X, Y), d(X, Z)\} .
$$

LEMMA 3. A necessary and sufficient condition for the path $B_{1} B_{2} \cdots B_{n}$ to be a minimal spanning tree is that

$$
d\left(B_{i}, B_{k}\right) \geq d\left(B_{j}, B_{j+1}\right) \quad \text { for all } 1 \leq i \leq j<k \leq n .
$$

PROOF. The proof follows immediately from a well-known $[\mathbf{1}]$ condition for a tree to be a minimal spanning tree. It is also easily seen if all inequalities are strict

Received by the editors May 13, 1983 and, in revised form, September 6, 1984.

1980 Mathematics Subject Classification. Primary 51M05; Secondary 05C05, 90B99, 94C15. 


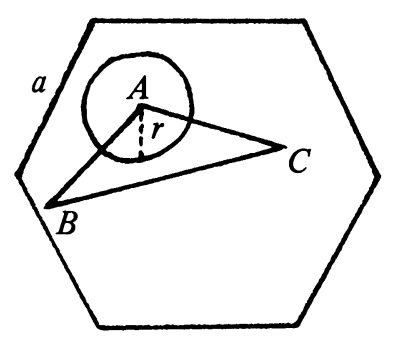

FIGURE 1. A regular hexagon containing the union of a triangle and a circle (except for the degenerate case $i=j=k-1$ ), then the path is "the" minimal spanning tree.

LEMMA 4. Suppose that $B_{1} B_{2} \cdots B_{n}, n \geq 3$, is a Steiner path and $B_{1} B_{2}, \ldots$, $B_{n} B_{1}$ form a convex polygon. Then $n \leq 7$ and $\varangle B_{1}+\varangle B_{n}=60 n-120$.

PROOF.

$$
\varangle B_{1}+\varangle B_{n}=(n-2) 180-\sum_{i=2}^{n-1} \varangle B_{i}=(n-2) 180-(n-2) 120=60 n-120 .
$$

Now $n \leq 7$ follows from the assumption of convex polygon.

The following lemma will be critically needed in proving the main results.

LEMMA 5. Let $H$ denote a regular hexagon of side a (the length). Let $R$ denote the region which consists of an isosceles triangle $\triangle A B C$ with $d(A, B)=d(A, C)$, and a circle with center $A$ and radius $r$ (see Figure 1). Suppose that $R$ is contained in $H$ and $d(B, C) \geq a$. Then

$$
d(A, B) \leq \sqrt{(\sqrt{3} a-r)^{2}+(a / 2)^{2}} \equiv z .
$$

PROOF. Let $h_{1}, \ldots, h_{6}$ denote the six corners of $H$. Let $H^{\prime}$ be a smaller regular hexagon with the six corners $h_{1}^{\prime}, \ldots, h_{6}^{\prime}$ such that $H^{\prime}$ and $H$ are concentric and the corresponding sides are parallel with distance $r$ (see Figure 2).

Then Lemma 5 can be restated as follows:

"Show that for any isosceles triangle $\triangle A B C, d(A, B)=d(A, C)$, satisfying the conditions that (i) $A$ is in $H^{\prime}$, (ii) $B$ and $C$ are in $H$, (iii) $d(B, C) \geq a$, we have $d(A, B) \leq z\left(=d\left(g^{\prime}, h_{3}\right)\right.$ where $g^{\prime}$ is the midpoint of $\left.h_{5}^{\prime} h_{6}^{\prime}\right)$."

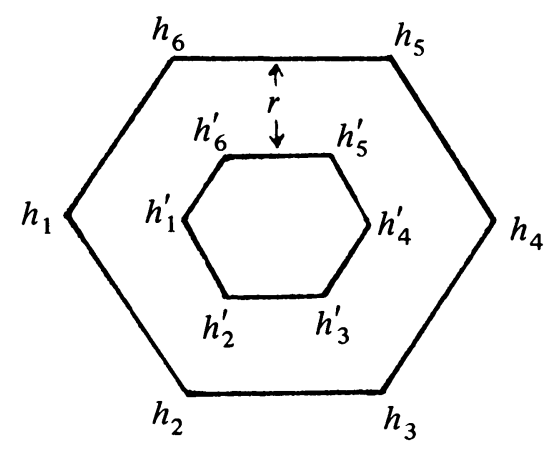

FIGURF 2. Two concentric regular hexagons 
We may assume without loss of generality that $A$ lies on the boundary of $H^{\prime}$ and at least one of $B$ and $C$ lies on the boundary of $H$. By symmetry we may further assume that $A$ lies on $g^{\prime} h_{5}^{\prime}$. Let $g$ be the midpoint of $h_{2} h_{3}$ and $g^{\prime \prime}$ the midpoint of $h_{1} h_{2}$. It is easy to see that the distances from $g^{\prime}$ to $h_{i}, i=1, \ldots 6$, and the distances from $h_{5}^{\prime}$ to $g, h_{3}, h_{4}, h_{5}, h_{6}, h_{7}$ and $g^{\prime \prime}$ are all at most $z$. Thus by Lemma 2 , the distance from $g^{\prime}$ to any point in $H$ and the distance from $h_{5}^{\prime}$ to any point in the heptahedron $F=g^{\prime \prime} g h_{3} h_{4} h_{5} h_{6} h_{1} g^{\prime \prime}$ is at most $z$. Applying Lemma 2 once more, we see that the distance from $A$ to any point in $F$ is at most $z$. Thus the only points $B$ and $C$ which can violate the lemma must fall in the triangle $g h_{2} g^{\prime \prime}$. The diameter of this triangle, however, is at most $d\left(g, g^{\prime \prime}\right)<a$, and so the lemma is proven.

3. The main results. Let $A=A_{1}, \ldots, A_{n}$ be a set of points on a unit circle with center $O$. Define

$$
m=\min \left\{\left[\alpha \beta+\sqrt{\alpha^{2}+\left(1-\beta^{2}\right) / 4}\right] /\left(\alpha^{2}+1 / 4\right), \gamma\right\}
$$

where $\alpha=\sqrt{3}+1-1 /(2 \rho), \beta=1-(1-\rho) \pi / \rho, \gamma=2(\sqrt{3}+1) /\left[(\sqrt{3}+1)^{2}+1 / 4\right]=$ $.70832 \ldots$ and $\rho$ is the Steiner ratio.

THEOREM. If the polygon $P_{n}=A_{1} A_{2} \cdots A_{n}$ has at most one side longer than $m$, then the Steiner minimal tree for $A$ is the sides of $P_{n}$ excluding the longest one.

ProOF. By Lemma 3 the minimal spanning tree for $A$ is the sides of $P_{n}$ excluding the longest one. We now prove that a Steiner minimal tree for $A$ cannot have a Steiner point.

Suppose to the contrary that $T$ is a Steiner minimal tree for $A$ and $T$ contains a Steiner point. It is well known [4] that the edges of a Steiner minimal tree can be uniquely decomposed at points of $A$ into nonempty components in which every $A_{i}$ is of degree 1 and is connected to a Steiner point if there is one in the component. Decompose $T$ into such components $T_{1}, T_{2}, \ldots$. Then there exists a $T_{i}$ containing a Steiner point. Now $T_{i}$ partitions $P_{n}$ into convex regions $Q_{1}, \ldots, Q_{q}$. Choose that region $Q_{w}$ containing the center $O$ of the circle. The boundary $P_{w}$ of $Q_{w}$ is made up of a subpath $A_{i}, A_{i+1}, \ldots, A_{j}$ of the polygon $P_{n}$ and a Steiner path $A_{i}=s_{0}, s_{1}, \ldots, s_{k}, s_{k+1}=A_{j}$, where $s_{1}, \ldots, s_{k}$ are Steiner points of $T$. Define

$$
\begin{gathered}
u_{w}=d\left(s_{c}, s_{c+1}\right)=\max _{l=0, \ldots, k} d\left(s_{l}, s_{l+1}\right), \\
v_{w}=d\left(A_{t}, A_{t+1}\right)=\max _{l=i, \ldots, j-1} d\left(A_{l}, A_{l+1}\right) .
\end{gathered}
$$

Then $v_{w} \geq u_{w}$, since otherwise we could substitute the appropriate edge of the path $A_{i}, \ldots, A_{j}$ for the edge $s_{c} s_{c+1}$ and shorten the length of $T$. Also, we have $s_{c} s_{c+1}$ appearing in at least one other region $Q_{w^{\prime}}$, where again $d\left(s_{c}, s_{c+1}\right) \leq u_{w^{\prime}} \leq v_{w^{\prime}}$. But since at most one of $v_{w}$ and $v_{w^{\prime}}$ is greater than $m$, then

$$
u_{w} \leq \min \left\{v_{w}, v_{w^{\prime}}\right\} \leq m .
$$

We now construct a regular hexagon $H$ with vertices, $h_{1}, \ldots, h_{6}$ and side $u_{w}$ such that $s_{m}, m=\lceil k / 2\rceil(\lceil x\rceil$ denotes the smallest integer not less than $x)$, is a vertex of $H$ and the two edges $\left[s_{m-1}, s_{m}\right]$ and $\left[s_{m}, s_{m+1}\right]$ overlap the sides of $H$. Then $P_{w}$ is contained in $H$ (see Figure 3 ). 


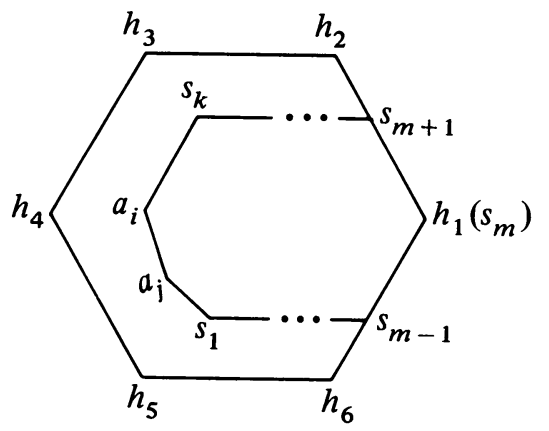

FIGURE 3. A regular hexagon containing $P_{w}$

We first consider the case that the center $O$ is not in the polygon $P_{w}^{\prime}=A_{j} s_{1} \ldots$ $s_{k} A_{i}$. Then $P_{w}^{\prime}$ is completely contained in the region enclosed by $A_{1} A_{j}$ and $A_{i} A_{j}$, where $A_{i} A_{j}$ denote the shorter of the two arcs from $A_{i}$ to $A_{j}$. If $d\left(A_{i}, A_{j}\right)<\sqrt{3}$, then $A_{i} A_{j}<120^{\circ}$. But this is impossible since if $k=1$, then $\varangle A_{i}+\varangle A_{j}<60$, and if $k>1$, then $\varangle A_{i}+\varangle A_{j}<60^{\circ}+60^{\circ}=120^{\circ}$, both contradicting Lemma 4 .

If $d\left(A_{i}, A_{j}\right) \geq \sqrt{3}$, then

$$
2 m \geq 2 u_{w} \geq d\left(A_{i}, A_{j}\right) \geq \sqrt{3} \text { by Lemma } 1
$$

implies that $m \geq \sqrt{3} / 2>.708$, a contradiction to $\gamma<1$.

Next we consider the case that center $O$ lies in the polygon $P_{w}^{\prime}$. Let $D$ be the point on the Steiner path $A_{j} s_{1} \cdots s_{k} A_{i}$ closest to $O$ and let $d(D, O)=r$. Note that the circle with center $O$ and radius $r$, as well as the triangle $\triangle O A_{i} A_{j}$, is contained in $H$. Furthermore, $d\left(A_{i}, A_{j}\right) \geq d\left(A_{t}, A_{t+1}\right)=v_{w} \geq u_{w}$. Hence the conditions of Lemma 5 are satisfied and we conclude

$$
1=d\left(O, A_{i}\right) \leq \sqrt{\left(\sqrt{3} u_{w}-r\right)^{2}+\left(u_{w} / 2\right)^{2}} \leq \sqrt{(\sqrt{3} m-r)^{2}+(m / 2)^{2}}
$$

or equivalently,

$$
r \leq \sqrt{3} m-\sqrt{1-(m / 2)^{2}}
$$

If $1-r<m$, then we have

$$
1-m<r \leq \sqrt{3} m-\sqrt{1-(m / 2)^{2}}
$$

or

$$
m>2(\sqrt{3}+1) /\left[(\sqrt{3}+1)^{2}+1 / 4\right]=\gamma,
$$

a contradiction to the definition of $m$.

If $1-r \geq m$, partition $T$ into $T^{\prime}$ and $T^{\prime \prime}$ at $D$. Let $A$ be partitioned into $A^{\prime}$ and $A^{\prime \prime}$ accordingly. Then $T^{\prime}$ and $T^{\prime \prime}$ are Steiner minimal trees for the point-sets $A^{\prime} \cup\{D\}$ and $A^{\prime \prime} \cup\{D\}$, respectively. Let $L(t)$ denote the length of the tree $t$. Let $p$ denote the perimeter of $P_{n}$ and let $a$ and $b$ denote the longest and the second longest side of $P_{n}$. Note that $b \leq m \leq 1-r$. Furthermore, consider $\triangle A_{i} O D$ for each $A_{i} \in A$. Then we have $d\left(A_{i}, D\right) \geq d\left(O, A_{i}\right)-d(O, D)=1-r \geq b$. It follows that

$$
L_{M}\left(A^{\prime} \cup\{D\}\right)+L_{M}\left(A^{\prime \prime} \cup\{D\}\right) \geq p-a-b+2(1-r) .
$$


Therefore,

$$
\begin{aligned}
p-a & \geq L(T)=L\left(T^{\prime}\right)+L\left(T^{\prime \prime}\right) \\
& \geq \rho L_{M}\left(A^{\prime} \cup\{D\}\right)+\rho L_{M}\left(A^{\prime \prime} \cup\{D\}\right) \geq \rho[p-a-b+2(1-r)],
\end{aligned}
$$

or

$$
\begin{aligned}
2 \rho(1-r) & \leq(1-\rho)(p-a)+\rho b \leq(1-\rho)(p-b)+\rho b \\
& \leq(1-\rho) p+(2 \rho-1) b<(1-\rho) 2 \pi+(2 \rho-1) m .
\end{aligned}
$$

Hence,

$$
r>1-(1-\rho) \pi / \rho-(2 \rho-1) m /(2 \rho) .
$$

But we have shown that $r \leq \sqrt{3} m-\sqrt{1-(m / 2)^{2}}$. Combining these two inequalities, we obtain

$$
\alpha m-\beta>\sqrt{1-(m / 2)^{2}}
$$

where $\alpha$ and $\beta$ are defined at the beginning of this section (note that $\alpha>1 / 2$ and $\beta<1$ ). Solving for $m$, we obtain

$$
m>\left[\alpha \beta+\sqrt{\alpha^{2}+\left(1-\beta^{2}\right) / 4}\right] /\left(\alpha^{2}+1 / 4\right),
$$

or

$$
m<\left[\alpha \beta-\sqrt{\alpha^{2}+\left(1-\beta^{2}\right) / 4}\right] /\left(\alpha^{2}+1 / 4\right)<0,
$$

both lead to a contradiction to the definition of $m$. We have thus proved that $T$ cannot have a Steiner point.

Substituting $\rho$ by .8 in the Theorem, we obtain

COROLlaRY. The Steiner minimal tree for $A$ is the sides of $P_{n}$ excluding the longest one if there is at most one side longer than $.55762 \ldots$

4. Some concluding remarks. Graham [5] conjectured that if $A$ is a set of points on a circle such that the largest angle formed by two consecutive points at the center is at most $\pi / 3$, then no Steiner minimal tree for $A$ contains a Steiner point. The updated version of the conjecture is that at most one such angle can be larger than $\pi / 3$. If the conjecture is stated using side rather than angle, then the condition becomes that at most one side can be longer than the radius. In a private communication Graham informed us that he can use the "probe" idea given in [3] to prove this conjecture if the degree $\pi / 3$ is replaced by $20^{\circ}$, which corresponds to a side of length .35 assuming radius one. Thus the result of this paper can be used to improve on the length .35 significantly. On the other hand, we note that $m$ is at most $.70832 \ldots$ even if one can prove the conjecture of Gilbert and Pollak that $\rho=\sqrt{3} / 2$. Therefore, the approach in this paper is not strong enough to prove the conjecture of Graham.

ACKNOWLEDGEMENT. The authors wish to thank a referee for very helpful comments.

\section{REFERENCES}

1. W. M. Boyce, M. R. Garey and D. S. Johnson, A note on bisecting minimal spanning trees, Networks 8 (1978), 187-192.

2. D. Z. Du and F. K. Hwang, A new bound for the Steiner ratio, Trans. Amer. Math. Soc. 278 (1983), 137-148. 
3. M. R. Garey, R. L. Graham and D. S. Johnson, The complexity of computing Steiner minimal trees, SIAM J. Appl. Math. 32 (1977), 835-859.

4. E. N. Gilbert and H. O. Pollak, Steiner minimal trees, SIAM J. Appl. Math. 16 (1968), 1-29.

5. R. L. Graham, Some results on Steiner minimal trees, unpublished manuscript.

6. J. B. Kruskal, On the shortest spanning subtree of a graph, Proc. Amer. Math. Soc. 7 (1956), 48-50.

7. R. C. Prim, Shortest connecting networks and some generalizations, Bell System Tech. J. 36 (1957), 1389-1401.

ACAdemia Sinica, Beijing, China

AT\&T Bell Laboratories, Murray Hill, NeW Jersey 07974

North EAst Heavy Machinery Institute, QIQIHaER, China 